\title{
Amanita ahmadii, a new species of Amanita subgenus Amanitina section Validae from Pakistan
}

\author{
Sana Jabeen ${ }^{1,2}$, Munazza Kiran², Junaid Khan ${ }^{3}$, Ishtiaq Ahmad ${ }^{1,4,5}$, \\ Habib Ahmad ${ }^{5}$, Hassan Sher ${ }^{3}$, Abdul Nasir Khalid ${ }^{2}$
}

I Department of Botany, Division of Science and Technology, University of Education, Lahore, Punjab, Pakistan 2 Department of Botany, University of the Punjab, Quaid-e-Azam Campus-54590, Lahore, Punjab, Pakistan 3 Center for Plant Sciences and Biodiversity, University of Swat, Swat, Khyber Pakhtunkhwa, Pakistan 4 Centre of Plant Biodiversity and Conservation, University of Peshawar, Peshawar, Khyber Pakhtunkhwa, Pakistan 5 Islamia College University, Peshawar, Khyber Pakhtunkhwa, Pakistan

Corresponding author: Sana Jabeen (sanajabeenue@gmail.com; sanajabeen@ue.edu.pk)

Academic editor: M.-A. Neves | Received 21 November 2018 | Accepted 26 December 2018 | Published 23 July 2019

Citation: Jabeen S, Kiran M, Khan J, Ahmad I, Ahmad H, Sher H, Khalid AN (2019) Amanita ahmadii, a new species of Amanita subgenus Amanitina section Validae from Pakistan. MycoKeys 56: 81-99. https://doi.org/10.3897/ mycokeys. 56.31819

\begin{abstract}
A new species from coniferous forests in Pakistan, Amanita ahmadii, is described on the basis of morphoanatomy and molecular data set analyses. This species is characterized by its medium-sized to large basidiomata, grayish brown to brown pileal surface and rimose pileus margin with gray to dark brown verrucose veil remnants, a cream stipe with bulbous base having grayish brown or brown longitudinal striations above the annulus, a scaly surface towards the base, globose to broadly ellipsoid and amyloid basidiospores, and the absence of clamped septa in all tissues. Molecular phylogenetic analyses based on ITS and LSU sequences confirmed its identity as a new taxon nested within subgen. Amanitina sect. Validae.
\end{abstract}

\section{Keywords}

Amanitaceae, nrDNA, Swat

Copyright Sana Jabeen et al. This is an open access article distributed under the terms of the Creative Commons Attribution License (CC BY 4.0), which permits unrestricted use, distribution, and reproduction in any medium, provided the original author and source are credited. 


\section{Introduction}

Amanitaceae E. J. Gilbert is a large family of agaricoid fungi that has been classified by many mycologists and split into various genera subgenera and sections (Corner and Bas 1962; Bas 1969). During recent years, it has been split into two genera, Amanita Pers., a genus of putatively ectomycorrhizal fungi, and Saproamanita Redhead, Vizzini, Drehmel \& Contu, a genus of putatively saprotrophic fungi (Redhead et al. 2016). This generic split has been rejected by Tulloss et al. (2016) based in part on the guidelines of Vellinga et al. (2015) for introducing new genera. Concise amended characterizations have been provided for the monophyletic family Amanitaceae and its two monophyletic genera, Amanita and Limacella Earle. This declaration is based on the current use of next-generation sequencing in studies of fungal ecology opposing the splitting of the genus. Recently Cui et al. (2018) and Yang et al. (2018) inferred the phylogeny of Amanitaceae based on multi-locus sequencing data. The results indicated that Amanitaceae is monophyletic and consists of five genera. The genus Amanita consists of $95 \%$ of the species which are characterized by agaricoid basidiomata, colorless and hyaline, ballistosporic and smooth basidiospores, free lamellae, presence of volval remnants (Persoon 1797). A total of 540 known species of Amanita are distributed worldwide (Yang 2000, Kirk et al. 2008, Menolli et al. 2009, Tulloss 2009, Wartchow et al. 2009, Justo et al. 2010, Wartchow and Gamboa-Trujillo 2012, Cho et al. 2015, Hosen et al. 2015, Tang et al. 2015, Wartchow and Cortez 2016, Jabeen et al. 2017, Cui et al. 2018, Kiran et al. 2018a, b). From Pakistan, 19 species of Amanita are known to date (Ahmad et al. 1997, Jabeen et al. 2017, Kiran et al. 2018a, b). Tulloss et al. (2001) described one new species, $A$. pakistanica Tulloss, S.H. Iqbal \& Khalid, but refrained from describing two more due to lack of materials. The work on these species is in progress by several workers, and it is estimated that the total number of Amanita from Pakistan could be above 50. Many taxa of the genus have been reported as edibles (Tulloss and Bhandary 1992, Buyck 1994, Montoya-Esquivel 1997), though some others are deadly poisonous (Yang 2015, Cai et al. 2016). Most of the species are ecologically important forming mycorrhizal symbiosis (Yang 1997, 2000, Kiran et al. 2018a).

Members of Amanita subgen. Amanitina (E. J. Gilbert) E. J. Gilbert have nonstriated pileus margins, attenuate lamellulae and amyloid basidiospores (Cui et al. 2018)). Six sections in this subgenus are recognized (Cui et al. 2018), based on the morphology of the remnants of the universal veil and the pileal margin. The sect. Validae is characterized by pilei that are usually distinctly colored, margins that are non-appendiculate and do not exceed the gill margin, non-fragile and membranous annuli and basal bulbs that are usually small (Tulloss and Yang 2018, Yang 1997, Cui et al. 2018).

During our ongoing studies of ectomycorrhizal fungi in Khyber Pakhtunkhwa province, we collected specimens of an unknown Amanita species belonging to Amanita subgen. Amanitina sect. Validae. The aim of the present study was to characterize 
and identify the taxon based on molecular phylogeny using the sequence data of the internal transcribed spacer (ITS) and partial large subunit (LSU) of ribosomal RNA. Here, we describe this taxon as a new species.

\section{Materials and methods}

\section{Sampling sites}

Specimens were collected from three different areas in two districts of Khyber Pakhtunkhwa province of Pakistan. One of these, the Swat district, has a very rich biodiversity. The mountains are covered with snow throughout the winter and in summer temperature ranges between $16-33^{\circ} \mathrm{C}$. The average annual precipitation in Swat district ranges from $1000 \mathrm{~mm}$ to $1200 \mathrm{~mm}$. The first area, Gabin Jabba, is a lush green valley in Swat district, which is characterized by a moist temperate vegetation with Picea smithiana (Wall.) Boiss. and Abies pindrow Royle as the dominant tree species. Mashkun, the second area in Swat district, is in the western part of the Himalayas. This collection site is a dry temperate forest with A. pindrow, P. smithiana and Cedrus deodara (Roxb. ex D. Don) G. Don as the dominant tree species along with Pinus wallichiana A. B. Jacks.

The third area is Kumrat valley, which lies at the extreme North of the Dir Upper district. It is located in the foothills of the Hindu Kush mountains with an elevation of about 950-2440 m (Siddiqui et al. 2013). Snowfall occurs frequently in winter, rainfall during monsoon season ranges from $100 \mathrm{~mm}$ to $255 \mathrm{~mm}$. Forests are dominated by a mixture of $C$. deodara, A. pindrow, Picea smithiana, and Pinus wallichiana, and Populus nigra L. is the main broad-leaved tree.

\section{Macroscopic and microscopic characterization}

Specimens were collected during routine macrofungal surveys and photographed in their natural habitats using a Nikon D3200 camera. Morphological features of fresh specimens were recorded and colors were designated using Munsell Soil Color Charts (Munsell 1975) and then forced-air dried for long term preservation. For detailed anatomical descriptions, tissues from different parts of the basidiomata were mounted on glass slides in 5\% Potassium Hydroxide solution $(\mathrm{KOH}$; w/v). Phloxine $(1 \% \mathrm{w} / \mathrm{v}$ aqueous solution) was used for a better contrast. Melzer's reagent was used to check the amyloidity of basidiospores. Anatomical features were noted under a compound microscope (MX4300H, Meiji Techno Co., Ltd, Japan). Measurements were recorded using a Carl Zeiss (Jena) ocular micrometer and line drawings were made using Leitz Wetzlar camera lucida. Size and shape of basidiospores are presented in a form following the description of ranges for biometric variables according to Tulloss (2016). Voucher specimens are deposited in the Herbarium at the University of the Punjab (LAH), Quaid-e-Azam Campus, Lahore, Pakistan and at the Swat University Herbarium (SWAT), Swat, Pakistan. 


\section{DNA extraction, PCR and sequencing}

For genomic DNA extraction, a standard CTAB method (Bruns 1995) was followed. Internal transcribed spacer regions along with central $5.8 \mathrm{~S}$ region of nuclear ribosomal DNA (nrDNA) were amplified (Gardes and Bruns 1993) using forward primer ITS1F and reverse primer ITS4 (White et al. 1990). For LSU amplification, LR0R as forward and LR5 as reverse primers were used (Ge et al. 2014). The PCR products were sent to Macrogen Inc. (Korea) for sequencing.

\section{Sequence alignment and phylogenetic analyses}

Consensus sequences were generated from the sequences obtained by both primers (forward and reverse) in BioEdit software v. 7.2.5 (Hall 1999). Sequences of Amanita subgen. Amanitina sect. Validae at NCBI (http://www.ncbi.nlm.nih.gov/) and from published literature (Kim et al. 2013, Cai et al. 2014, Cui et al. 2018) were added to the datasets. Taxa from the sect. Phalloideae were chosen as outgroup (Cui et al. 2018). Shorter ITS and LSU sequences were omitted from the final matrices. Species and specimens used for the molecular phylogenetic analyses are given in Table 1. Multiple sequences were aligned using online webPRANK by EMBL-EBI, Wellcome Trust Genome Campus, UK (https://www.ebi.ac.uk/goldman-srv/webprank/). The phylogeny was inferred by maximum likelihood (ML) analysis using model selection for best DNA analysis for each dataset in MEGA6 software (Tamura et al. 2013). Models with the lowest BIC scores (Bayesian Information Criterion) were considered to describe the substitution pattern the best. Non-uniformity of evolutionary rates among sites may be modeled by using a discrete gamma distribution $(+\mathrm{G})$ with 5 rate categories and by assuming that a certain fraction of sites are evolutionarily invariable $(+\mathrm{I})$. The phylogenetic analyses were performed at 1000 bootstrap replicates. Percentage identity and divergence in nrDNA-ITS of the taxa were analyzed using MegAlign (DNAStar, Inc.). Sequences generated in this study were submitted to GenBank under accession numbers KY996724, KY996755, MF116158 and MF070490 for ITS and KY996725 and MK166021 for LSU.

\section{Results}

\section{Phylogeny}

Consensus sequences of the ITS region were BLAST searched at NCBI. These sequences showed $98 \%$ identity to $A$. aff. fritillaria (KJ466372 and KJ466373) sequences from China (Cai et al. 2014) with $94-100 \%$ query cover. It also showed $95 \%$ identity with an A. franchetii (JX515561) sequence from Bulgaria with 100\% query cover and $0.0 \mathrm{E}$ value. The LSU consensus sequence BLAST at NCBI showed $99 \%$ identity to A. aff. flavoconia (HQ539663) and Amanita sp. (KT072738) sequences from the eastern USA and $A$. fritillaria (KF245897) sequences from South Korea with 99\% query cover. 
Table I. Species and specimens of Amanita used for the molecular phylogenetic analyses.

\begin{tabular}{|c|c|c|c|c|c|}
\hline \multirow[t]{2}{*}{ Species } & \multirow[t]{2}{*}{ Voucher } & \multirow[t]{2}{*}{ Country } & \multicolumn{2}{|c|}{ GenBank accession number } & \multirow[t]{2}{*}{ Reference } \\
\hline & & & ITS & LSU & \\
\hline A. aff. brunnescens & BW_HF 10C & USA & - & HQ539661 & - \\
\hline \multirow[t]{2}{*}{ A. aff. citrina } & BW_PNC & USA & - & HQ539662 & - \\
\hline & HKAS 34170 & China & AY436449 & AY436489 & $\begin{array}{l}\text { Zhang et al. } 2004 \text {, } \\
\text { Thongbai et al. } 2016\end{array}$ \\
\hline \multirow[t]{2}{*}{ A. aff. flavorubens } & PSMCC 121 & USA & - & HQ539663 & - \\
\hline & BW_HF-FR & USA & - & HQ539664 & - \\
\hline \multirow[t]{2}{*}{ A. aff. fritillaria } & HKAS56832 & China & KJ466372 & KJ466479 & $\begin{array}{c}\text { Cai et al. 2014, Thongbai } \\
\text { et al. } 2016\end{array}$ \\
\hline & HKAS57649 & China & KJ466373 & KJ466480 & Cai et al. 2014 \\
\hline A. aff. spissacea & $2 \mathrm{C} 5$ & Japan & AB973749 & - & - \\
\hline \multirow[t]{4}{*}{ A. ahmadii } & LAH35010 & Pakistan & KY996724 & KY996725 & - \\
\hline & SWAT0001351 & Pakistan & MF070490 & - & - \\
\hline & LAH35241 & Pakistan & KY996755 & MK166021 & - \\
\hline & LAH35242 & Pakistan & MF116158 & - & - \\
\hline \multirow[t]{2}{*}{ A. augusta } & DBB49390 & USA & JQ937287 & & - \\
\hline & DBB21873 & USA & JX515564 & - & - \\
\hline A. augusta as " $A$. franchetii" & 07040 & USA & GQ250398 & - & - \\
\hline A. bisporigera & RET 377-9 & USA & KJ466374 & KJ466434 & Thongbai et al. 2016 \\
\hline A. brunneolocularis & ANDES_F313 NVE57 & Colombia & FJ890033 & FJ890044 & Vargas et al. 2011 \\
\hline \multirow[t]{6}{*}{ A. brunnescens } & RET 637-7 & USA & KT006762 & KT006766 & Thongbai et al. 2016 \\
\hline & BW_HP12 & USA & - & HQ539674 & - \\
\hline & RET 529-10 & USA & KP284273 & KP284284 & - \\
\hline & RET 554-1 & USA & KP284275 & KP284285 & - \\
\hline & RET 549-9 & USA & - & KP284283 & - \\
\hline & JS94/2 & - & - & AF097379 & Drehmel et al. 1999 \\
\hline A. castanea & MFLU 15-1424 & Thailand & KU904823 & KU877539 & Thongbai et al. 2016 \\
\hline A. cf. flavorubescens & JMP0098 & USA & EU819454 & - & Palmer et al. 2008 \\
\hline \multirow[t]{2}{*}{ A. cf. spissacea } & BZ2015-40 & Thailand & KY747464 & - & Cai et al. 2012 \\
\hline & OR1214 & Thailand & KY747469 & KY747478 & Cai et al. 2012 \\
\hline \multirow[t]{9}{*}{ A. citrina } & LEM 960298 & Japan & AB015679 & - & $\begin{array}{l}\text { Oda et al. 1999, } \\
\text { Thongbai et al. } 2016\end{array}$ \\
\hline & JM96/61 & - & - & AF097378 & - \\
\hline & TM02_102 & Canada & - & EU522722 & Porter et al. 2008 \\
\hline & KA12-1226 & South Korea & KF245908 & KF245892 & Kim et al. 2013 \\
\hline & JSH s.n. & - & - & AF041547 & - \\
\hline & JS94/1 & - & - & AF097377 & Drehmel et al. 1999 \\
\hline & ANDES_F405 IP25 & Colombia & - & FJ890046 & Vargas et al. 2011 \\
\hline & BW JLR 102106-1 & USA & - & HQ539679 & - \\
\hline & KA12-1612 & South Korea & KF245909 & KF245893 & Kim et al. 2013 \\
\hline \multirow[t]{6}{*}{ A. citrinoindusiata } & HKAS100522 & China & MH508320 & MH486468 & Cui et al. 2018 \\
\hline & HKAS58884 & China & MH508323 & MH486471 & Cui et al. 2018 \\
\hline & HKAS58886 & China & MH508324 & MH486472 & Cui et al. 2018 \\
\hline & HKAS58796 & China & MH508321 & MH486469 & Cui et al. 2018 \\
\hline & HKAS58888 & China & MH508325 & MH486473 & Cui et al. 2018 \\
\hline & HKAS58874 & China & MH508322 & MH486470 & Cui et al. 2018 \\
\hline \multirow[t]{2}{*}{ A. excelsa } & HKAS 31510 & Germany & AY436453 & AY436491 & Thongbai et al. 2016 \\
\hline & Ge 816 & China & - & HQ539691 & - \\
\hline
\end{tabular}




\begin{tabular}{|c|c|c|c|c|c|}
\hline \multirow[t]{2}{*}{ Species } & \multirow[t]{2}{*}{ Voucher } & \multirow[t]{2}{*}{ Country } & \multicolumn{2}{|c|}{ GenBank accession number } & \multirow[t]{2}{*}{ Reference } \\
\hline & & & ITS & LSU & \\
\hline \multirow[t]{3}{*}{ A. flavipes } & KA12-0685 & South Korea & KF245911 & KF245895 & Kim et al. 2013 \\
\hline & HKAS 36582 & China & AY436455 & & Zhang et al. 2004 \\
\hline & KA12-1517 & South Korea & KF245912 & KF245896 & Kim et al. 2013 \\
\hline \multirow[t]{6}{*}{ A. flavoconia } & TENN61564 & USA & JF313655 & - & - \\
\hline & BW_PH22 & - & - & HQ539693 & - \\
\hline & ANDESF408CV3 & Colombia & FJ890029 & FJ890041 & Thongbai et al. 2016 \\
\hline & TM03_435 25S & Canada & - & EU522816 & Porter et al. 2008 \\
\hline & NVE 351 & Colombia & KF937301 & - & Vasco-Palacios et al. 2014 \\
\hline & NVE 242 & Colombia & KF937300 & - & Vasco-Palacios et al. 2014 \\
\hline \multirow[t]{2}{*}{ A. flavoconia } & HKAS 34047 & USA & AY436456 & & Zhang et al. 2004 \\
\hline & RV5Aug96 & - & - & AF042609 & Moncalvo et al. 2000 \\
\hline A. flavorubens & RET 295-9 & USA & - & HQ539694 & - \\
\hline \multirow{3}{*}{ A. flavorubescens } & TENN61660 & USA & JF313650 & - & - \\
\hline & F:PRL6062 & USA & GQ166902 & - & Thongbai et al. 2016 \\
\hline & RV96/102 & - & - & AF097380 & Drehmel et al. 1999 \\
\hline A. franchetii & JM96/27 & - & - & AF097381 & Drehmel et al. 1999 \\
\hline \multirow{3}{*}{$\begin{array}{l}\text { A. franchetii f. lactella as } \\
\text { "A. franchetii" }\end{array}$} & DBBJUS01 & Spain & JX515563 & - & - \\
\hline & DBB52095 & Bulgaria & JX515562 & - & - \\
\hline & DBB51482 & Bulgaria & JX515561 & - & - \\
\hline $\begin{array}{l}\text { A. franchetii f. queletii as } \\
\text { "A. aspera" }\end{array}$ & IFO-8262 & - & AF085485 & - & Lim and Jung 1998 \\
\hline \multirow[t]{3}{*}{ A. fritillaria } & - & China & JF273505 & - & Legendre et al. 2009 \\
\hline & HKAS 38331 & China & AY436457 & - & Zhang et al. 2004 \\
\hline & KA12-1231 & South Korea & KF245913 & KF245897 & Kim et al. 2013 \\
\hline A. lavendula & RET 639-7 & USA & KP866163 & KR865979 & Thongbai et al. 2016 \\
\hline A. luteofusca & PSC $1093 \mathrm{~b}$ & Australia & - & HQ539705 & - \\
\hline A. luteolovelata & PSC 2187 & Australia & - & HQ539706 & - \\
\hline \multirow[t]{3}{*}{ A. morrisii } & RET 672-6 & USA & KR919762 & KR919770 & - \\
\hline & RET 271-7 & USA & KT213441 & KT213442 & Thongbai et al. 2016 \\
\hline & RET 445-10 & USA & KR919760 & KR919768 & - \\
\hline \multirow[t]{6}{*}{ A. novinupta } & GO-2009-234 & Mexico & KC152066 & - & - \\
\hline & GO-2009-315 & Mexico & KC152065 & - & - \\
\hline & GO-2009-301 & Mexico & KC152067 & - & - \\
\hline & RET 060-2 & USA & KF561974 & KF561978 & Thongbai et al. 2016 \\
\hline & RET 093-10 & USA & - & HQ539716 & - \\
\hline & NY 00066710 & USA & KJ535437 & KJ535441 & - \\
\hline A. phalloides & GDGM:40312 & Italy & KC755034 & - & \\
\hline \multirow[t]{7}{*}{ A. porphyria } & LEM960303 & Japan & AB015677 & - & Oda et al. 1999 \\
\hline & DAVFP:26784 & USA & JF899548 & - & \\
\hline & RET 079-1 & Switzerland & KP866181 & KP866192 & Thongbai et al. 2016 \\
\hline & HKAS 31531 & China & AY436471 & AY436500 & Thongbai et al. 2016 \\
\hline & RET 309-8 & Norway & KP866176 & KP866189 & - \\
\hline & RET 404-2 & $\begin{array}{c}\text { Czech } \\
\text { Republic }\end{array}$ & KP866171 & KP866184 & - \\
\hline & RET 404-9 & $\begin{array}{c}\text { Czech } \\
\text { Republic }\end{array}$ & - & KP866185 & - \\
\hline
\end{tabular}




\begin{tabular}{|c|c|c|c|c|c|}
\hline \multirow[t]{2}{*}{ Species } & \multirow[t]{2}{*}{ Voucher } & \multirow[t]{2}{*}{ Country } & \multicolumn{2}{|c|}{ GenBank accession number } & \multirow[t]{2}{*}{ Reference } \\
\hline & & & ITS & LSU & \\
\hline \multirow[t]{15}{*}{ A. rubescens } & JMP0003 & USA & EU819464 & - & Palmer et al. 2008 \\
\hline & TRTC156957 & Canada & JN020972 & - & Dentinger et al. 2011 \\
\hline & LE241998 & Russia & JF313652 & - & - \\
\hline & RK01-01 & Denmark & AJ889923 & - & - \\
\hline & EMF4 & China & JF273507 & - & - \\
\hline & LEM950063 & Japan & $\mathrm{AB} 015682$ & - & Oda et al. 1999 \\
\hline & ASIS23255 & South Korea & KM052530 & - & - \\
\hline & ASIS23444 & South Korea & KM052535 & - & - \\
\hline & KA $12-1221$ & Korea & KF245919 & KF245903 & Thongbai et al. 2016 \\
\hline & RET $122-8$ & Turkey & - & HQ539735 & - \\
\hline & ANDES_F416 NVE160 & Colombia & FJ890031 & FJ890043 & Vargas et al. 2011 \\
\hline & RV5Aug96 & - & - & AF042607 & Moncalvo et al. 2000 \\
\hline & RV97/23 & - & - & AF097383 & Drehmel et al. 1999 \\
\hline & JM96/53 & - & - & AF097382 & Drehmel et al. 1999 \\
\hline & KA12-0936 & South Korea & KF245918 & KF245902 & Kim et al. 2013 \\
\hline \multirow[t]{6}{*}{ A. sp. } & ANDES_F241 IP24 & Colombia & FJ890032 & FJ890047 & Vargas et al. 2011 \\
\hline & RET 516-10 & USA & KP711830 & KP711838 & - \\
\hline & RET 516-5 & USA & KP711836 & KP711837 & - \\
\hline & RET 530-1 & USA & KT072736 & КT072737 & - \\
\hline & RET 539-8 & USA & KT072735 & KT072738 & - \\
\hline & HKAS 38419 & China & AY436474 & AY436502 & Thongbai et al. 2016 \\
\hline \multirow[t]{5}{*}{ A. spissa } & UP541 & - & EF493270 & - & Nygren et al. 2008 \\
\hline & KF02-47 & - & AJ889924 & - & - \\
\hline & UP542 & - & EF493271 & - & Nygren et al. 2008 \\
\hline & KA12-0884 & South Korea & KF245910 & KF245894 & Kim et al. 2013 \\
\hline & NYBG 47779 & Germany & - & HQ539743 & - \\
\hline \multirow[t]{6}{*}{ A. spissacea } & LEM960187 & Japan & $\mathrm{AB} 015683$ & - & Oda et al. 1999 \\
\hline & ASIS24872 & South Korea & KM052552 & KU139485 & - \\
\hline & ASIS26240 & - & KT894841 & KU139454 & - \\
\hline & ASIS24978 & - & KM052550 & KU139487 & - \\
\hline & ASIS24775 & - & KM052543 & KU139484 & - \\
\hline & ASIS24949 & - & KM052546 & KU139486 & - \\
\hline \multirow[t]{3}{*}{ A. virosa } & HKAS 56694 & China & JX998030 & JX998058 & Cai et al. 2012 \\
\hline & HMJAU23304 & China & KJ466431 & KJ466498 & Cai et al. 2012 \\
\hline & JM 97/42 & - & - & AF159086 & Moncalvo et al. 2000 \\
\hline
\end{tabular}

Taxa from subgen. Amanitina sect. Phalloideae (Fr.) Quél. were chosen as the outgroup (Kim et al. 2013). The sequences generated during this study clustered with the similar taxa in sect. Validae (Figs 1-3). Our species clustered with $A$. aff. fritillaria, $A$. citrinoindusiata, A. franchetii f. franchetii, A. franchetii f. lactella (as $A$. franchetii in GenBank), A. franchetii f. queletii (as $A$. aspera in GenBank) and A. spissa in phylogenetic analysis. However, $A$. ahmadii separated from $A$. aff. fritillaria with a strong bootstrap value of $95 \%, 49 \%$ and $100 \%$ in ITS, LSU and ITS+LSU sequence dataset analyses, respectively (Figs 1-3). 


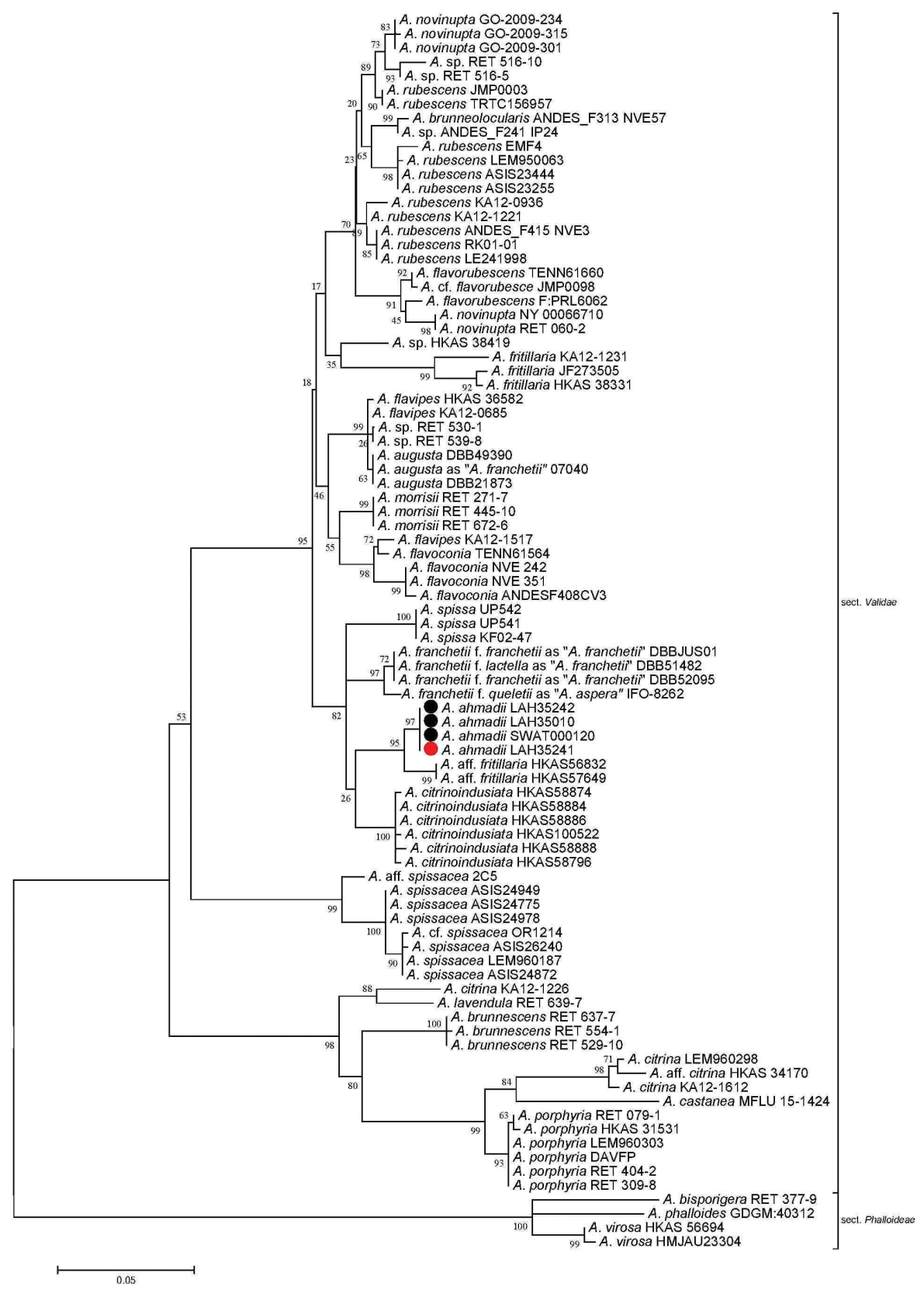

Figure I. Molecular phylogenetic analysis of ITS sequences using the maximum likelihood method based on the Tamura 3-parameter model (Tamura 1992). The percentage of trees in which the associated taxa clustered together is shown next to the branches. A discrete gamma distribution was used to model evolutionary rate differences among sites $(5$ categories $(+G$, parameter $=0.4454))$. The tree is drawn to scale, with branch lengths measured in the number of substitutions per site. The analysis involved $88 \mathrm{nu}-$ cleotide sequences. There were a total of 1018 positions in the final dataset. Sequences generated during the present investigation are marked with bullets. Red represents the holotype. 


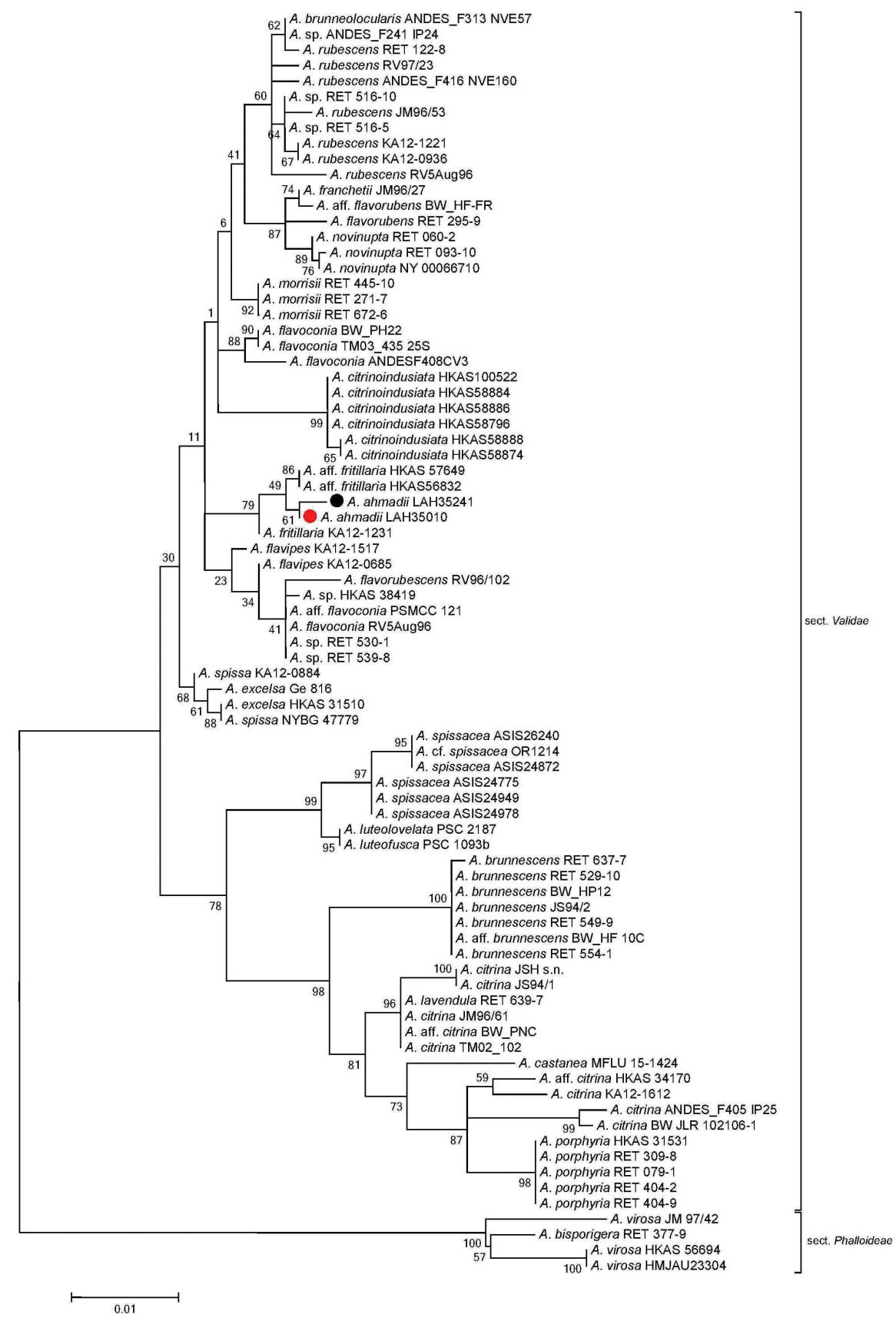

Figure 2. Molecular phylogenetic analysis of LSU sequences by using the maximum likelihood method based on the Kimura 2-parameter model (Kimura 1980). A discrete gamma distribution was used to model evolutionary rate differences among sites $(5$ categories $(+G$, parameter $=0.2164))$. The tree is drawn to scale, with branch lengths measured in the number of substitutions per site. The analysis involved 81 nucleotide sequences. There were a total of 871 positions in the final dataset. Sequences generated during the present investigation are marked with bullets. Red represents the holotype. 


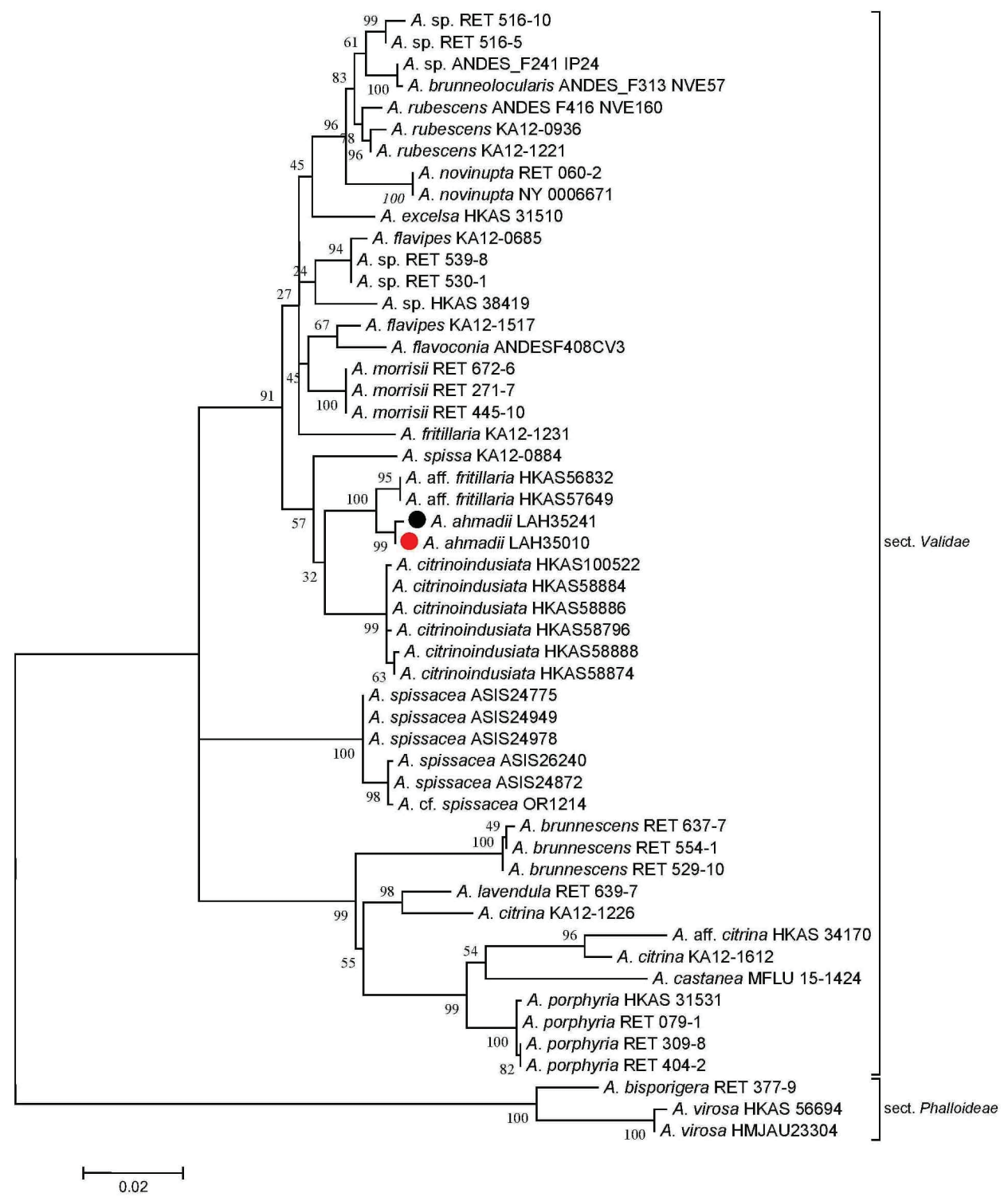

Figure 3. Molecular phylogenetic analysis of ITS+LSU sequences by using the maximum likelihood method based on the Tamura-Nei model (Tamura and Nei 1993). A discrete gamma distribution was used to model evolutionary rate differences among sites $(5$ categories $(+G$, parameter $=0.2250)$ ). The rate variation model allowed for some sites to be evolutionarily invariable $([+I], 43.3848 \%$ sites). The tree is drawn to scale, with branch lengths measured in the number of substitutions per site. The analysis involved 52 nucleotide sequences. There were a total of 1760 positions in the final dataset. Sequences generated during the present investigation are marked with bullets. Red represents the holotype. 


\section{Taxonomy}

Amanita abmadii Jabeen, I. Ahmad, Kiran, J. Khan \& Khalid, sp. nov. MycoBank number: MB821204

Figs 4,5

Diagnosis. Small to medium-sized basidiomata, grayish brown to brown pileal surface having rimose and non-appendiculate pileal margins, verrucose, gray to dark bluish or brown veil remnants, dry and split stipe surface at the base forming scales, globose to subglobose, smooth, amyloid basidiospores.

Holotype. Pakistan, Khyber Pakhtunkhwa province, Malakand division, Swat district, Mashkun, 2500 m a.s.l., on soil under Cedrus deodara, 5 Sept. 2013, Sana Jabeen SJ35 (LAH35010; GenBank ITS: KY996724; LSU: KY996725).

Etymology. The species epithet ahmadii refers to Sultan Ahmad, the pioneer Pakistani mycologist.

Description. Pileus 4-7 cm in diameter, convex to flat at maturity; cuticle gray (2.5BG4/2) to grayish brown (10YR3/2) or brown (2.5Y4/4) with time; surface dry; universal veil remnants on pileus verrucose, aligned in one direction, scattered, gray (2.5Y4/2) to dark brown (2.5Y2/2); margins non-appendiculate, incurved when young, highly rimose by maturity. Lamellae off-white (2.5BG4/2) to cream (5Y9/4) becoming brownish when dry, adnexed, subdistant to close; edges entire. Lamellulae small (1/3 of the lamellae), attenuate, truncate. Stipe $6.7-9 \times 0.6-1.5 \mathrm{~cm}$, apex slightly wider and white, with up to $1.5 \mathrm{~cm}$ wide bulbous base, central, cylindrical; surface with grayish brown $(5 \mathrm{GY} 5 / 2)$ striations above the annulus, splitting towards the base forming scales on white $(2.5 \mathrm{BG} / 2)$ to cream (5Y9/4) context. Annulus superior, membranous, skirtlike, with longitudinal striations on the upper surface, gray (2.5Y4/2) with a darker lower part. Universal veil absent. Ordorless and not changing color upon bruising.

Basidiospores [60/3/3] (6.5) 7-8.5 (9.5) × (6) 6.5-7.5 (8) $\mu \mathrm{m}, \mathrm{Q}=$ (1) 1.03-1.22 (1.33), avg $\mathrm{Q}=1.10$, globose to broadly ellipsoid, amyloid in Melzer's reagent. Basidia (32) 34.5-59 (67) $\times 7-8 \mu \mathrm{m}$, clavate, frequently 4 sterigmate, 2 sterigmata also observed, thin-walled, hyaline in $5 \% \mathrm{KOH}$. Subhymenium pseudoparenchymatous, cells isodiameteric, intermixed and densely packed. Veil remnants made up of hyphae with terminal subglobose to elongated cells (42.5) 49.5-54 (57) $\times(13) 13-16(19) \mu \mathrm{m}$ on a branched filament 3-4 $\mu \mathrm{m}$ wide; septa frequent; clamp connections absent. Pileipellis filamentous, 4-5 $\mu \mathrm{m}$ in diameter, branched, septate; clamp connections absent, light brown with some hyaline tissue in $5 \% \mathrm{KOH}$. Universal veil remnants of globose to subglobose cells (6.8) 8-12.2 (12.7) $\times(4.4) 7.5-10.5$ (11) $\mu \mathrm{m}$ with filaments (0.7) $0.9-2.6(3.5) \mu \mathrm{m}$ in diameter. Hyphae from stipe 3-24 $\mu \mathrm{m}$ wide, filamentous, branched, hyaline in $5 \% \mathrm{KOH}$, septate; clamp connections absent in all tissues.

Habitat and distribution. In coniferous forests of Pakistan with a moist temperate to dry temperate climate. 


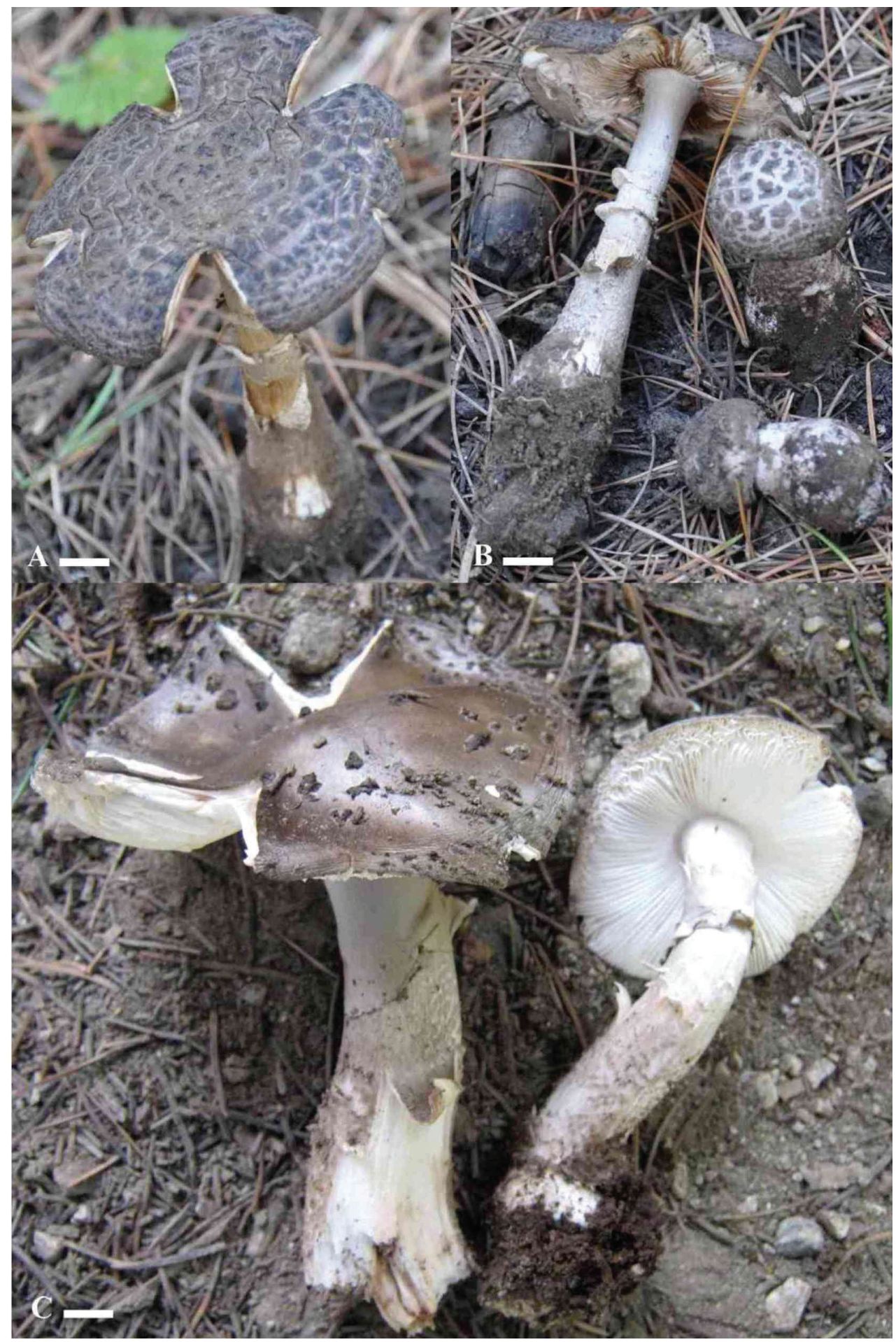

Figure 4. Amanita ahmadii basidiomata. A, B LAH35010 (holotype) C SWAT0001351. Photos by Abdul Nasir Khalid and Junaid Khan. Scale bars: $1 \mathrm{~cm}(\mathbf{A}) ; 1.2 \mathrm{~cm}(\mathbf{B}) ; 0.5 \mathrm{~cm}(\mathbf{C})$. 


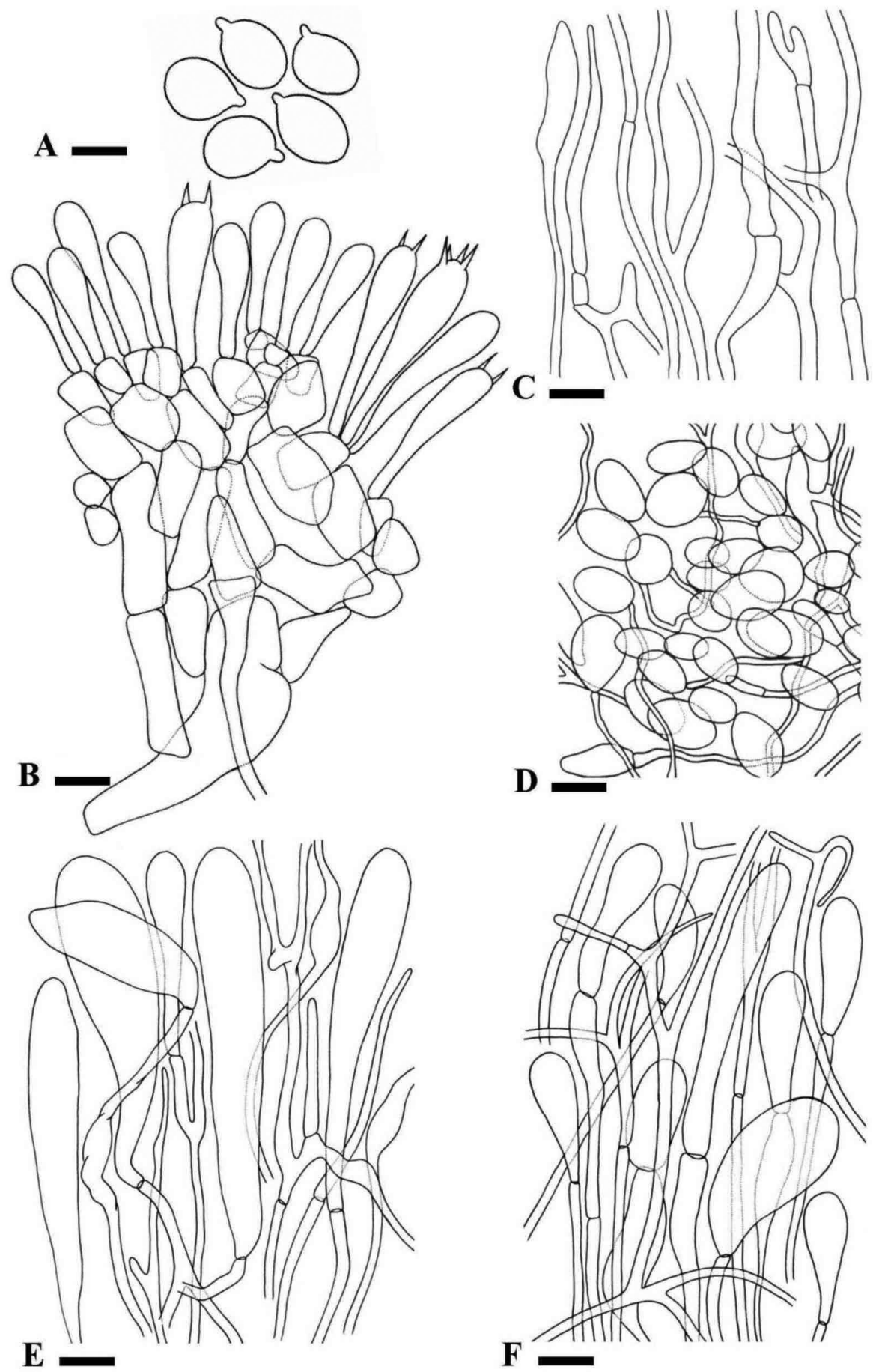

Figure 5. Amanita ahmadii LAH35010 (holotype). A Basidiospores B Basidia, basidioles and subhymenium C Pileipellis D Universal veil remnants on pileus surface $\mathbf{E}$ Hyphae from stipe $\mathbf{F}$ Partial veil. Drawings by Sana Jabeen. Scale bars: $5.5 \mu \mathrm{m}(\mathbf{A}) ; 8 \mu \mathrm{m}$ (B-D); $22 \mu \mathrm{m}(\mathbf{E}, \mathbf{F})$. 
Additional specimens examined. Pakistan, Khyber Pakhtunkhwa province, Malakand division, Dir Upper district, Kumrat, $2232 \mathrm{~m}$ a.s.l., on soil under conifers, 2 Sept. 2015, Abdul Nasir Khalid FS82 (LAH35241; GenBank ITS: KY996755; LSU: MK166021); Swat district, Mashkun, 2500 m a.s.l., on soil under Cedrus deodara, 4 Aug. 2013, Ishtiaq Ahmad IS213P65 (LAH35242; GenBank ITS: MF116158); Gabin Jabba valley, $2450 \mathrm{~m}$ a.s.l., on soil under Picea smithiana, 30 Aug. 2015, Junaid Khan GJ-1508 (SWAT001351; GenBank ITS: MF070490).

\section{Discussion}

Amanita ahmadii is characterized by its grayish brown to brown pileus surface with abundant gray to dark brown verrucose veil remnants and by its rimose margins. Anatomically it is characterized by its globose to broadly ellipsoid basidiospores. The species is morphologically similar to A. fritillaria Sacc. by its grayish to brownish gray pileus surface, and verrucose volval remnants. Amanita fritillaria differs by bearing ellipsoid basidiospores (Corner and Bas 1962, Yang 1997, 2005, 2015). In phylogenetic trees based on ITS, LSU and combined sequence datasets of both regions, A. fritillaria was inferred as a distinct lineage from $A$. ahmadii.

Amanita aff. fritillaria (HKAS56832 and HKAS57649, Cai et al. 2014) forms a sister clade to A. ahmadii (Figs 1-3), but it is morphologically distinct. The former taxon possesses a brownish and purplish pileus surface (Zhu L. Yang pers. comm.) while the latter has a grayish brown or brown pileus surface with highly rimose margins (Cai et al. 2014). Amanita fritillaria f. malayensis Corner \& Bas was described from Singapore (Corner and Bas 1962), but more recently was also found in subtropical, evergreen, broad-leaved forests in China; it differs from $A$. ahmadii in having a dark umber to rather pale grayish umber pileus (Yang 2005, 2015).

The European sequences labeled as " $A$. franchetii" and " $A$. aspera" in GenBank are close relatives of $A$. ahmadii in the ITS phylogenetic analysis. Amanita franchetii (Boud.) Fayod is somewhat variable in appearance and there are three morphological infraspecific taxa, including $A$. franchetii f. franchetii (Boud.) Fayod (JX515562 and JX515563), A. franchetii f. lactella Neville \& Poumarat (JX515561) and A. franchetii f. queletii (Bon \& Dennis) Neville \& Poumarat (AF085485) (Neville and Poumarat 2004). The last taxon most closely resembles $A$. ahmadii but differs in having more yellow hues on the stipe and pronounced reddening on the bulb with age. Amanita augusta Bojantchev \& R. M. Davis, as "A. franchetii" in GenBank (GQ250398), another species from western North America looks similar to $A$. ahmadii but its yellowish brown pileus with yellow universal veil remnants and ellipsoid spores (Bojantchev and Davis 2013) distinguishes it from $A$. ahmadii. During phylogenetic analyses, all these taxa were inferred as distinct species.

The novel species also showed differences from $A$. castanea Thongbai, Tulloss, Raspé $\&$ K. D. Hyde from Thailand. Amanita castanea bears a viscid, shiny and sericeous pileal surface, which is dark brown at center and light brown to brownish orange towards 
margin, with universal veil mostly towards the margin, rarely over disc, as scattered gray to brownish gray, reddish brown to grayish brown warts or small floccose patches and globose basidiospores (Thongbai et al. 2016). All these characters distinguish $A$. castanea from $A$. ahmadii. In molecular phylogenetic analyses, $A$. castanea is clustered with the species in a distant clade within sect. Validae (Figs 1-3). Amanita ahmadii also showed morphological distinctions from $A$. citrinoindusiata Zhu L. Yang, Y. Y. Cui \& Q. Cai, a newly reported species in the same section from China. This species is characterized by its robust, brownish gray, gray to dark gray pileus and a stipe bearing a citrine to yellowish annulus. This suggests it is a separate species from $A$. ahmadii (Cui et al. 2018). Molecular data also supports the separation of these two taxa in phylogenetic trees (Figs 1-3).

The European $A$. excelsa Gonn. \& Rabenh is also morphologically close to $A$. ahmadii in having a gray-brown pileus. However, $A$. excelsa differs from $A$. ahmadii in having mealy, gray irregular and non-persistent patches of volval remnants on the pileus. The volva in $A$. excelsa has 2-5 pale ochre brown zones of friable material above the bulb, and lastly, the broadly ellipsoid to ellipsoid, occasionally elongate basidiospores also distinguish $A$. excelsa from $A$. ahmadii (Neville \& Poumarat, 2004). The phylogenetic position of these taxa also indicates that they are separate. Based on morphological characters and molecular phylogenic analysis, our new species belongs to Amanita subgen. Amanitina sect. Validae.

\section{Acknowledgements}

This work was financially supported by the Higher Education Commission (HEC)Pakistan to Dr Sana Jabeen under Indigenous PhD Fellowships for 5000 Scholars (Phase-II), Dr Sana Jabeen, Dr Ishtiaq Ahmad and Munazza Kiran under International Research Support Initiative Program (IRSIP) and Dr Hassan Sher under PakUS science and technology promotion program. We sincerely thank Prof. Donald H. Pfister for providing the opportunity to Dr Sana Jabeen, Dr Ishtiaq Ahmad and Munazza Kiran to work in his laboratory at Department of Organismic and Evolutionary Biology, Harvard University, MA, USA. Thanks are also due to Prof. Dr Zhu-Liang Yang (Key Laboratory for Plant Diversity and Biogeography of East Asia, Kunming Institute of Botany, Chinese Academy of Sciences, Kunming, China) for providing pictures to compare the morphology of the specimens and review of the manuscript. Special thanks are due to Dr Else C. Vellinga, (Department of Plant and Microbial Biology, University of California, Berkeley, CA, USA) for editing the text. Her useful comments and suggestions greatly improved this article. Authors are grateful to Dr Rosanne Healy (Assistant Scientist, Department of Plant Pathology, University of Florida. Gainesville, FL 32611) for linguistic suggestions and helpful comments resulting in the removal of technical errors. We are also thankful to Dr Abdul Rehman Khan Niazi (Department of Botany, University of the Punjab, Lahore, Pakistan) and all laboratory fellows for accompanying the tours to different areas of Pakistan. 


\section{References}

Ahmad S, Iqbal SH, Khalid AN (1997) Fungi of Pakistan. Sultan Ahmad Mycological Society Pakistan, 248 pp.

Bas C (1969) Morphology and subdivision of Amanita and a monograph of its section Lepidella. Persoonia 5: 285-579.

Bojantchev D, Davis RM (2013) Amanita augusta, a new species from California and the Pacific Northwest. North American Fungi 8: 1-11. https:/doi.org/10.2509/naf2013.008.005

Bruns TD (1995) Thoughts on the processes that maintain local species diversity of ectomycorrhizal fungi. In: Collins HP, Robertson GP, Klug MJ (Eds) The Significance and Regulation of Soil Biodiversity. Developments in Plant and Soil Sciences 63. Springer, Dordrecht, 63-73. https://doi.org/10.1007/978-94-011-0479-1_5

Buyck B (1994) Ubwoba: Les champignons comestibles de l'ouest du Burundi. Administration generale de la cooperation au developpement, Publication Agricole 34, Bruxelles, 123 pp.

Cai Q, Tang LP, Yang ZL (2012) DNA Barcoding of economically important mushrooms: A case study on lethal amanitas from China. Plant Diversity and Resources 34(6): 614-622. https://doi.org/10.3724/SP.J.1143.2012.12140

Cai Q, Tulloss RE, Tang LP, Tolgor B, Zhang P, Chen ZH, Yang ZL (2014) Multi-locus phylogeny of lethal amanitas: implications for species diversity and historical biogeography. BMC Evolutionary Biology 14: 143-158. https://doi.org/10.1186/1471-2148-14-143

Cai Q, Cui YY, Yang ZL (2016) Lethal Amanita species in China. Mycologia 108: 993-1009. https://doi.org/10.3852/16-008

Cho H, Park MS, Lee H, Oh SY, Jang Y, Fong JJ, Lim YW (2015) Four new species of Amanita in Inje County, Korea. Mycobiology 43: 408-414. https://doi.org/10.5941/ MYCO.2015.43.4.408

Corner EJH, Bas C (1962) The genus Amanita in Singapore and Malaya. Persoonia 2: 241-304.

Cui YY, Cai Q, Tang LP, Liu JW, Yang ZL (2018) The family Amanitaceae: molecular phylogeny, higher-rank taxonomy and the species in China. Fungal Diversity 91(1): 5-230. https://doi.org/10.1007/s13225-018-0405-9

Dentinger BT, Didukh MY, Moncalvo JM (2011) Comparing COI and ITS as DNA barcode markers for mushrooms and allies (Agaricomycotina). PLoS ONE 6(9): e25081. https:// doi.org/10.1371/journal.pone.0025081

Drehmel D, Moncalvo JM, Vilgalys R (1999) Molecular phylogeny of Amanita based on largesubunit ribosomal DNA sequences: implications for taxonomy and character evolution. Mycologia 91(4): 610-618. https://doi.org/10.2307/3761246

Gardes M, Bruns TD (1993) ITS primers with enhanced specificity for basidiomycetes application to the identification of mycorrhizae and rusts. Molecular Ecology 2: 113-118. https:// doi.org/10.1111/j.1365-294X.1993.tb00005.x

Ge C, Cui YN, Jing PY, Hong XY (2014) An alternative suite of universal primers for genotyping in multiplex PCR. PloS ONE 9: e92826. https://doi.org/10.1371/journal.pone.0092826

Hall TA (1999) BioEdit: a user-friendly biological sequence alignment editor and analysis program for Windows 95/98/NT. Nucleic Acids Symposium Series 41: 95-98. 
Hosen MI, Li TH, Deng WQ (2015) Amanita cinereovelata, a new species of Amanita section Lepidella from Bangladesh. Mycological Progress 14: 35. https://doi.org/10.1007/s11557015-1058-7

Jabeen S, Kiran M, Ullah S, Wilson A, Mueller GM, Fiaz M, Khalid AN (2017) Amanita glarea, a new species in section Vaginatae from Pakistan. Phytotaxa 306: 135-145. https:// doi.org/10.11646/phytotaxa.306.2.3

Jukes TH, Cantor CR (1969) Evolution of protein molecules. In: Munro HN (Ed.) Mammalian Protein Metabolism. Academic Press, New York, 21-132. https://doi.org/10.1016/ B978-1-4832-3211-9.50009-7

Justo A, Morgenstern I, Hallen-Adams HE, Hibbett DS (2010) Convergent evolution of sequestrate forms in Amanita under Mediterranean climate conditions. Mycologia 102: 675-688. https://doi.org/10.3852/09-191

Kim CS, Jo JW, Kwag YN, Oh J, Shrestha B, Sung GH, Han SK (2013) Four newly recorded Amanita species in Korea: Amanita sect. Amanita and sect. Vaginatae. Mycobiology 41: 131-138. https://doi.org/10.5941/myco.2013.41.3.131

Kiran M, Khan J, Naseer A, Sher H, Khalid AN (2018a) Amanita pallidorosea in Pakistan and its ectomycorrhizal association with Quercus oblongata. Mycotaxon 132(4): 799-811. https://doi.org/10.5248/132.799

Kiran M, Khan J, Sher H, Pfister DH, Khalid AN (2018b) Amanita griseofusca: a new species of Amanita in section Vaginatae from Malam Jabba, Swat, Pakistan. Phytotaxa 364(2): 181-192. https://doi.org/10.11646/phytotaxa.364.2.5

Kirk PM, Cannon PF, Minter DW, Stalpers JA (Eds) (2008) Dictionary of the Fungi (10 ${ }^{\text {th }}$ edn). CABI International, Wallingford, $784 \mathrm{pp}$.

Legendre P, Mi X, Ren H, Ma K, Yu M, Sun IF, He F (2009) Partitioning beta diversity in a subtropical broad-leaved forest of China. Ecology 90(3): 663-674. https://doi. org/10.1890/07-1880.1

Lim YW, Jung HS (1998) Phylogenetic relationships of Amanita species based on ITS1-5.8 S rDNA-ITS2 region sequences. The Journal of Microbiology 36(3): 203-207.

Menolli N Jr, Capelari M, Baseia IG (2009) Amanita viscidolutea, a new species from Brazil with a key to Central and South American species of Amanita section Amanita. Mycologia 101: 395-400. https://doi.org/10.3852/07-079

Moncalvo JM, Lutzoni FM, Rehner SA, Johnson J, Vilgalys R (2000) Phylogenetic relationships of agaric fungi based on nuclear large subunit ribosomal DNA sequences. Systematic Biology 49(2): 278-305. https://doi.org/10.1093/sysbio/49.2.278

Montoya-Esquivel A (1997) Estudio etnomicológico en San Francisco Temezontla, estado de. Tlaxcala. Tesis de Maestría, Universidad Nacional Autonoma de Mexico, 133 pp. [In Spanish] Munsell (1975) Munsell Color Co - Munsell Soil Color Charts. Baltimore, MD, 20 pp.

Nei M, Kumar S (2000) Molecular Evolution and Phylogenetics. Oxford University Press, New York, $333 \mathrm{pp}$.

Neville P, Poumarat S (2004) Amaniteae. Vol. 1. Fungi Europaei 9: 1-1119.

Nygren CM, Eberhardt U, Karlsson M, Parrent JL, Lindahl BD, Taylor AF (2008) Growth on nitrate and occurrence of nitrate reductase-encoding genes in a phylogenetically di- 
verse range of ectomycorrhizal fungi. New Phytologist 180(4): 875-889. https://doi. org/10.1111/j.1469-8137.2008.02618.x

Oda T, Tanaka C, Tsuda M (1999) Molecular phylogeny of Japanese Amanita species based on nucleotide sequences of the internal transcribed spacer region of nuclear ribosomal DNA. Mycoscience 40(1): 57-64. https://doi.org/10.1007/BF02465674

Palmer JM, Lindner DL, Volk TJ (2008) Ectomycorrhizal characterization of an American chestnut (Castanea dentata) - dominated community in western Wisconsin. Mycorrhiza 19(1): 27. https://doi.org/10.1007/s00572-008-0200-7

Persoon CH (1797) Tentamen dispositionis methodicae fungorum. Petrum Philippum Wolf, Leipzig, Vol. 8, 1-76. http://bibdigital.rjb.csic.es/ing/Libro.php?Libro=3125

Porter TM, Skillman JE, Moncalvo J (2008) Fruiting body and soil rDNA sampling detects complementary assemblage of Agaricomycotina (Basidiomycota, Fungi) in a hemlockdominated forest plot in southern Ontario. Molecular Ecology 17(13): 3037-3050. https://doi.org/10.1111/j.1365-294X.2008.03813.x

Redhead SA, Vizzini A, Drehmel DC, Contu M (2016) Saproamanita, a new name for both Lepidella E.-J. Gilbert and Aspidella E.-J. Gilbert (Amanitae, Amanitaceae). IMA Fungus 7: 119-29. https://doi.org/10.5598/imafungus.2016.07.01.07

Tamura K (1992) Estimation of the number of nucleotide substitutions when there are strong transition-transversion and G+C-content biases. Molecular Biology and Evolution 9: 678687.

Tamura K, Nei M (1993) Estimation of the number of nucleotide substitutions in the control region of mitochondrial DNA in humans and chimpanzees. Molecular Biology and Evolution 10: 512-526.

Tamura K, Stecher G, Peterson D, Filipski A, Kumar S (2013) MEGA6: Molecular Evolutionary Genetics Analysis version 6.0. Molecular Biology and Evolution 30: 2725-2729. https://doi.org/10.1093/molbev/mst197

Tang LP, Cai Q, Lee SS, Buyck B, Zhang P, Yang ZL (2015) Taxonomy and phylogenetic position of species of Amanita sect. Vaginatae s.l. from tropical Africa. Mycological Progress 14: 1-15. https://doi.org/10.1007/s11557-015-1061-z

Thongbai B, Tulloss RE, Miller SL, Hyde KD, Chen J, Zhao RL, Raspé O (2016) A new species and four new records of Amanita (Amanitaceae; Basidiomycota) from northern Thailand. Phytotaxa 286(4): 211-231. https://doi.org/10.11646/phytotaxa.286.4.1

Tulloss RE (2009) Amanita magniverrucata - revision of an interesting species of Amanita section Lepidella. Mycotaxon 108: 93-104. https://doi.org/10.5248/108.93

Tulloss RE (2016) (mutable text) Biometric Variables: Meanings and How to Define a Range. Studies in the Amanitaceae. http://www.amanitaceae.org/?How\%20 To\%E2\%80\%99s\&howto=8 [Accessed on 2018-11-21]

Tulloss RE, Bhandary HR (1992) Amanita chepangiana - a new species from Nepal. Mycotaxon 43: 25-31.

Tulloss RE, Iqbal SH, Khalid AN, Bhatt RP, Bhat VK (2001) Studies in Amanita (Amanitaceae) from southern Asia. I. Some species of Pakistan's Northwest Frontier Province. Mycotaxon 77: 455-490. 
Tulloss RE, Yang ZL [Eds] (2018) Amanitaceae studies. http://www.amanitaceae.org [Accessed on: 2018-11-20]

Vargas N, Bernal A, Sarria V, Franco-Molano A, Restrepo S (2011) Amatoxin and phallotoxin composition in species of the genus Amanita in Colombia: a taxonomic perspective. Toxicon 58(6-7): 583-590. https://doi.org/10.1016/j.toxicon.2011.09.005

Vasco-Palacios AM, López-Quintero C, Franco-Molano AE, Boekhout T (2014) Austroboletus amazonicus sp. nov. and Fistulinella campinaranae var. scrobiculata, two commonly occurring boletes from a forest dominated by Pseudomonotes tropenbosii (Dipterocarpaceae) in Colombian Amazonia. Mycologia 106(5): 1004-1014. https://doi.org/10.3852/13-324

Wartchow F, Cortez VG (2016) A new species of Amanita growing under Eucalyptus is discovered in south Brazil. Mycosphere 7: 262-267. https://doi.org/10.5943/mycosphere/7/3/2

Wartchow F, Gamboa-Trujillo JP (2012) Amanita chocoana - a new species from Ecuador. Mycotaxon 121: 405-412. https://doi.org/10.5248/121.405

Wartchow F, Tulloss RE, Cavalcanti MA (2009) Amanita lippiae: a new species from the semiarid caatinga region of Brazil. Mycologia 101: 864-870. https://doi.org/10.3852/08-106

Yang ZL (1997) Die Amanita-Arten von Südwestchina. Bibliotheca Mycologica 170: 1-240.

Yang ZL (2000) Species diversity of the genus Amanita (Basidiomycetes) in China. Acta Botanica Yunnanica 22: 135-142.

Yang ZL (2005) Flora fungorum sinicorum. Vol. 27. Amanitaceae. Science Press, Beijing, $1-258$ [In Chinese]

Yang ZL (2015) Atlas of the Chinese species of Amanitaceae. Science Press, Beijing, 1-213 [In Chinese]

Yang ZL, Cai Q, Cui YY (2018) Phylogeny, diversity and morphological evolution of Amanitaceae. Biochemical Systematics and Ecology 34: 359-380.

Zhang LF, Yang JB, Yang ZL (2004) Molecular phylogeny of eastern Asian species of Amanita (Agaricales, Basidiomycota): taxonomic and biogeographic implications. Fungal Diversity 17: 219-238. 